\title{
A Direct Test on the Possibility of an Aggregate in Dispersion Being Disrupted by Shear Flow
}

\author{
Z. W. Sun, ${ }^{* 1}$ Y. M. Li, $\nmid$ S. H. Xu, $†$ L. R. Lou, $†$ G. L. Dai, ${ }^{*}$ and X. Q. Dong* \\ * National Microgravity Laboratory, Institute of Mechanics, Chinese Academy of Sciences, Beijing 100080, People's Republic of China; and $\dagger$ Open \\ Laboratory of Bond Selective Chemistry, University of Science and Technology of China, Hefei, 230026, Anhui, People's Republic of China
}

Received January 12, 2001; accepted June 8, 2001; published online August 16, 2001

\begin{abstract}
A direct test of the adhesive strength between particles $(2 \mu \mathrm{m}$ polystyrene latex spheres) in aggregate was carried out to probe the possibility of aggregates being disrupted by convection flows of certain shearing rates. Optical tweezers were used to manipulate individual particles or aggregates for tests in the flow field. The results of our experiment are analyzed to understand how shear flow affects the coagulation process. 2001 Academic Press
\end{abstract}

Key Words: coagulation; aggregate; polystyrene latex; shear flow; optical tweezers.

\section{INTRODUCTION}

In general, shear flow enhances the rate of collisions in a dispersion, but when viscous stresses due to shear flow exceed the attractive force between particles, existing aggregates may break up. Therefore, shear flow can affect the coagulation process in two ways: It can either accelerate or retard coagulation compared to the rate of coagulation due to Brownian motion alone. Which situation actually occurs is directly related to the nature of the particle-particle interactions (and, of course, particle concentration). For example, if the coagulation takes place at a second minimum of the interaction potential energy, then there will be a higher possibility that shear flow will only disrupt the dispersion's aggregates. In this case, the shear flow will slow the coagulation process.

There has been much effort devoted to the effect of shear on the coagulation process (1-4). Zeichner and Schowalter (1) used trajectory analysis to study the stability of colloidal dispersions in flow fields. They concluded that particulate dispersions can react in several different ways as the intensity of shearing is increased from zero: The dispersion can remain stable; it can redisperse if it has been initially flocculated into a weak secondary minimum of the interparticle potential curve; it can be flocculated into a strong primary minimum of the potential curve; or, in extreme cases, it can be redispersed from the primary minimum. However, their theoretical study has not yet been systematically verified by experiments.

\footnotetext{
${ }^{1}$ To whom correspondence should be addressed. Fax: 861062615524. E-mail: sunzw@mail.imech.ac.cn.
}

Information regarding the bond strength between particles against shear flow is essential to better understand the effect of shear on the coagulation process. Usually, the adhesive force measurements were performed for a sphere adhering to a plate (5-8). Until now, it had not been possible to directly measure the adhesive forces between real particles of a few micrometers in size or less (8). In the absence of a method to directly check the role of shear flow in the coagulation, sometimes its influence on the coagulation process has to be estimated or analyzed theoretically only. For example, Folkersma et al. $(9,10)$ compared their experiments of perikinetic coagulation for $2-\mu \mathrm{m}$ polystyrene lattices performed under microgravity $(\mu \mathrm{g})$ with experiments under regular 1- $g$ conditions and found that the coagulation rate under $\mu \mathrm{g}$ is 11.2 times greater than that under $1 \mathrm{~g}$. Furthermore, as they showed, their finding implies that it is gravity which makes the coagulation rate much (over one order of magnitude) lower than its theoretical value (von Smoluchowski value). They assumed that this profound difference in coagulation rates was caused by free convection, which was thought to be inevitable on the ground. According to their estimation, a shear rate value of $1.3 \mathrm{~s}^{-1}$ would be able to break up doublets. However, Sun and Qiao (11) found in their ground-based experiments that there was no noticeable difference in the coagulation rates observed whether (weak) convection flows existed or not. On the other hand, according to Folkersma's estimation in Ref. (10), very loosely bonded particles within aggregates could be disrupted by shear flow with a shear rate even as low as $1.3 \mathrm{~s}^{-1}$. At this point, a direct observation to test whether aggregates can be disrupted by flow at different shear levels under the same experimental conditions would be useful. Creating an optical trap (with optical tweezers) (12-19) makes such a test possible by allowing direct manipulation of individual particles or aggregates.

This work presents an experiment using an optical trap to directly test the possibility of a dispersion's aggregates being disrupted by convection flows and therefore provides an alternative outlook on the effect of shear flow.

\section{EXPERIMENTAL}

To make our experiment more compatible to the conditions of Refs. (9-11), the same sized (diameters of $2.013 \pm 0.025 \mu \mathrm{m}$ ), 


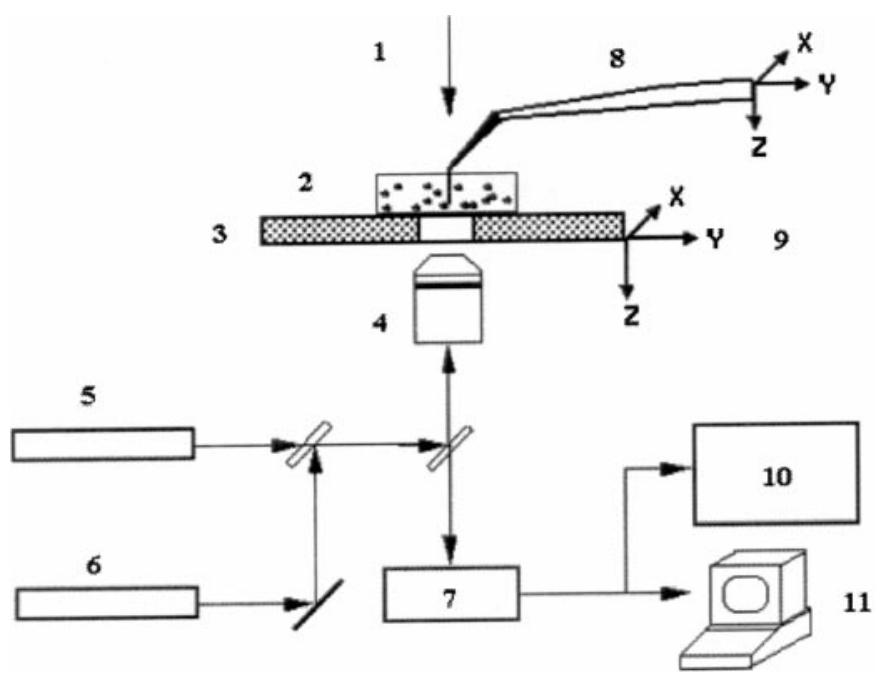

FIG . 1. Experimental setup. 1. illumination light; 2. sample cell; 3. sample stage; 4 . objective; 5 . light source of optical tweezers; 6 . light source of optical scalpel; 7. CCD camera; 8. glass object; 9. 3D motion; 10. video recorder; 11. computer.

monodispersed polystyrene (PS) lattices (product of Duke Scientific Corporation, USA) and the same electrolyte, $\mathrm{NaCl}$, with a concentration of $0.5 \mathrm{~mol} \cdot \mathrm{L}^{-1}$ (unless otherwise described) were used in this experiment.

The optical micromanipulating system used in our experiment is schematically shown in Fig. 1. It combines optical tweezers (trap) with an optical scalpel (microbeam). A diode pumped (continuous wave) $\mathrm{Nd}: \mathrm{YVO}_{4}$ laser is used as the light source of the optical tweezers. The $1.06 \mu \mathrm{m}$ laser beam from $\mathrm{Nd}$ : $\mathrm{YVO}_{4}$ is introduced into an inversed microscope, reflected by a dichromatic mirror, which is inserted in the microscope, and incident upon a high-numerical-aperture (NA of 1.35) oil-immersion microscope objective, which focuses a strongly convergent beam into a glass sample cell. Because of optical momentum transfer to the particle suspended in the solution in the cell, the strongly convergent beam will exert a force on the particle and hold the particle at a point near its focus. In this paper, the terms "trap" and "tweezers" represent the same thing. When used to hold or move a particle, the term "tweezers" is used. When talking about the active field, the term "trap" is used instead. An optical scalpel driven by a pulsed YAG: Nd laser source is also employed to break bonds between particles by using a single laser pulse.

The three-dimensional motion of the sample stage with an adjustable moving speed is controlled precisely by a computer. Manipulation of the trapped particle (or aggregate), that is, moving it relative to the sample cell or other particles, is accomplished by fixing the trapping laser beam and moving the microscope sample stage. A three-dimensional movable glass object was inserted into the sample cell for the aggregate to land on in the tests discussed in the following. An example of the view to be seen through the microscope system is shown in Fig. 2. Since some particles were not in the focusing plane, they look different in size and clarity although all single particles have the same size.

Aggregates were obtained for the tests in two ways: naturally and artificially. That is, aggregates were obtained naturally through the natural collisions of particles due to Brownian motion, or they were assembled artificially by means of the optical tweezers. To avoid the influence of the optical trapping force on the interaction between particles, in most cases we chose to use naturally formed aggregates.

An approximate evaluation of the magnitude and active range of the optical trapping force is necessary to understand its effect in the test. The Stokes hydrodynamic friction force exerted on a spherical particle in a fluid field is $f=6 \pi \eta a V$. Here $\eta$ is the viscosity of the liquid, $a$ is the radius of the particle, and $V$ is the flow speed toward the center of the spherical particle. To roughly estimate the optical trapping force holding a particle, we trapped a particle and then moved the liquid surrounding it. By gradually increasing the flow speed, we found a critical speed $V_{\mathrm{c}}$ at which the particle was washed away from the trapping area. We estimated the acting radius $R_{\mathrm{c}}$ for the optical trap by measuring how far away a particle started to move toward
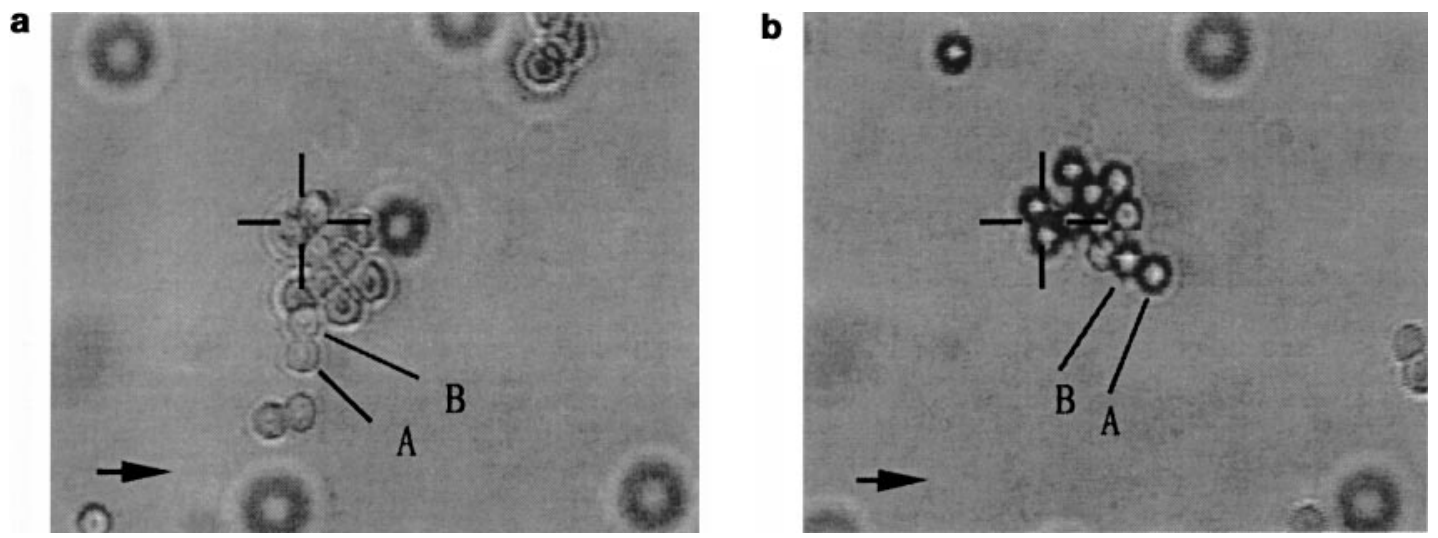

FIG . 2. The test for an aggregate in the flow field. The position of the trapping center is marked with + . (a) The case when the flow speed is zero. (b) The case when the flow speed is $15 \mu \mathrm{m} \cdot \mathrm{s}^{-1}$. 
the trapping center. Apparently, both $V_{\mathrm{c}}$ and $R_{\mathrm{c}}$ depend on the level of laser power driving the optical trap. In this experiment, the range of $R_{\mathrm{c}}$ is 1.5 to $\sim 2 \mu \mathrm{m}$. When the considered particle is connected to other particles, for example when a particle is a component of an aggregate, the hydrodynamic friction force $f$ exerted on it becomes $6 \pi \eta a V \chi$, where $\chi$ is a shielding coefficient (20) [or correction factor (21)], which should be taken into account when the considered particle has other particles nearby.

We conducted four types of experiments.

\section{Test 1}

We used an optical trap to hold an existing aggregate in solution, as shown in Fig. 2a (the trapping center is represented by "+"), and moved the stage with a speed of $15 \mu \mathrm{m} \cdot \mathrm{s}^{-1}$. Then the whole solution, except the aggregate held by the optical trap, moved with the stage. The flow direction with respect to the aggregate is shown in Fig. 2b. We can see that the hydrodynamic friction force made the aggregate rotate, but no particles peeled off. Apparently, holding a different part of the aggregate would result in its different final orientation. When we tried to increase the flow speed to $20 \mu \mathrm{m} \cdot \mathrm{s}^{-1}$, the whole aggregate moved away from the trapping area with the flow. Therefore, the hydrodynamic friction force caused by the $20-\mu \mathrm{m} \cdot \mathrm{s}^{-1}$ flow on the aggregate must have been larger than the trapping force.

Particle A located at the far end of the aggregate was beyond the influence of the trapping force ( $R_{\mathrm{c}}$ was $1.8 \mu \mathrm{m}$ for this test), and so this particle was chosen. Since liquid surrounding particle A was moving with speed $V=15 \mu \mathrm{m} \cdot \mathrm{s}^{-1}$, by taking the drag force on $\mathrm{A}$ to be equal to the hydrodynamic friction force caused by a shear flow (see Appendix), an equivalent shear rate $(\gamma \approx$ $V /[2 a \sin (\pi / 4)])$ for separation of this particle from its neighbor $\mathrm{B}$ can be estimated $\left(\approx 10.6 \mathrm{~s}^{-1}\right)$. This test showed that a shear rate of up to $10.6 \mathrm{~s}^{-1}$ was not enough to disrupt the aggregate.

\section{Test 2}

We moved the glass object to touch a chain-shaped aggregate until the aggregate adhered to the glass object, as shown in Fig. 3. While keeping the object in a fixed position, we tried to make the liquid move (by moving the sample cell) with respect to the aggregate. We increased the flow speed to $120 \mu \mathrm{m} \cdot \mathrm{s}^{-1}$ and tried different moving directions. Under the scour of flows, the aggregate was not disrupted and its original shape was well maintained. This test showed that under an equivalent shear rate of magnitude $85 \mathrm{~s}^{-1}$ there was still no disruption of the aggregate observed.

\section{Test 3}

Our experiment showed that when there was no $\mathrm{NaCl}$ added in the solution, particles were not able to stick together. When we brought them together artificially through an optical trap, they separated after the trapping force was turned off. Particles did not adhere to the wall or bottom of the sample cell either, unless

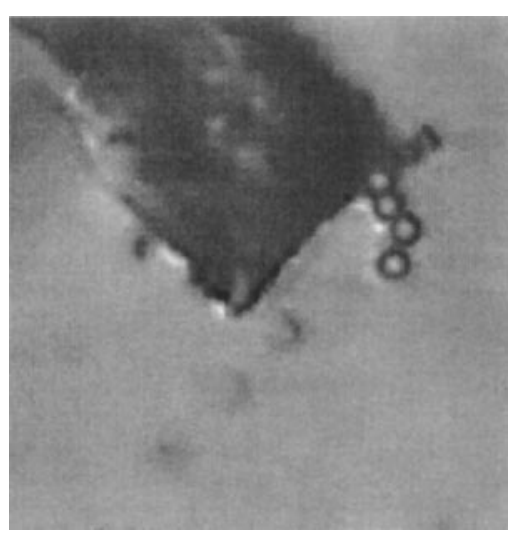

FIG. 3. The scouring test of flow to an aggregate adhered to a glass object in dispersion solution.

there was a high enough $\mathrm{NaCl}$ concentration $\left(>0.05 \mathrm{~mol} \cdot \mathrm{L}^{-1}\right)$ in the solution.

Particles adhered to the bottom or wall of the cell easily when $0.5 \mathrm{~mol} \cdot \mathrm{L}^{-1} \mathrm{NaCl}$ was maintained in the solution. We trapped one particle and put it on top of another one resting on the bottom, and then we put a third particle on top of the second. After this upright column was formed, we manipulated particles to adhere one after another to construct the letter " $X$ " centered on the top particle, as shown in Fig. 4a. The final " $X$ " structure was parallel to, but at a two-particle distance from, the cell bottom. These particles stuck together so firmly that we could not separate them with the optical tweezers.

Figure $4 \mathrm{~b}$ shows this " $\mathrm{X}$ " structure collapsing under the shock of a laser pulse from the optical scalpel. The magnitude of the energy transferred to the particles from the laser pulse is estimated to be about $10^{-10} \mathrm{~J}$.

\section{Test 4}

We moved one particle to touch another resting on the bottom of the cell. When the concentration of $\mathrm{NaCl}$ was $0.5 \mathrm{~mol} \cdot \mathrm{L}^{-1}$, the trapping force (even with the maximum available laser power in the experiment) was not strong enough to remove the first particle from its partner (Fig. 5a). This trapping force is roughly $2.4 \times 10^{-11} \mathrm{~N}$, according to the flow speed of $V=900 \mu \mathrm{m} \cdot \mathrm{s}^{-1}$ required to drive a particle away from the trap (how to use the Stokes formula to estimate this force is described in the next paragraph). When the above procedure was repeated under $0.3 \mathrm{~mol} \cdot \mathrm{L}^{-1} \mathrm{NaCl}$, we were able to remove the second particle (Fig. 5b). By gradually lowering the power level of the laser, and therefore the trapping force, we were able to find the minimum power level required to separate two particles sticking together. The magnitude of the trapping force can then be calibrated by a method similar to that described in Ref. (22). We used the optical tweezers, with the same (minimum) laser power level, to hold a single particle in the solution, and by adjusting the flow speed, the minimum required speed to wash a particle away from its trap was found to be $80 \mu \mathrm{m} \cdot \mathrm{s}^{-1}$. 
a

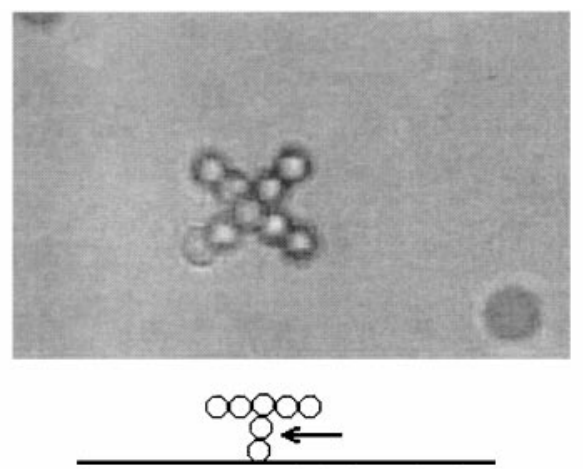

b

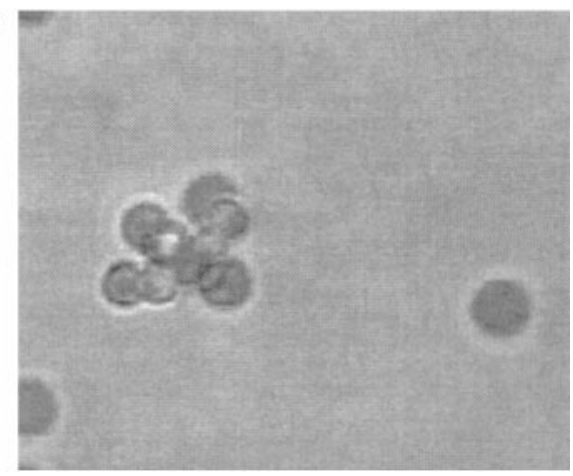

FIG . 4. "X" structure assembled artificially by means of the optical tweezers under $0.5 \mathrm{M} \mathrm{NaCl}$ concentration in solution. (a) These particles stuck together so firmly that we could not separate them with the optical tweezers. (b) "X" structure was collapsed by the shock of a laser pulse. The arrow underneath Fig. 4 a points to the target of the laser pulse.

Using the Stokes formula $f=6 \pi \eta a V$ with $a=1 \mu \mathrm{m}$ and $V=80 \mu \mathrm{m} \cdot \mathrm{s}^{-1}$, we can evaluate the adhesive force between particles to be $2 \times 10^{-12} \mathrm{~N}$ (under the concentration of $0.3 \mathrm{~mol}$. $\mathrm{L}^{-1} \mathrm{NaCl}$ used). To yield the same magnitude of a drag force in a shear flow, by using $f_{\gamma}=6 \pi \eta a(2 a) \gamma \chi \sin (\pi / 4) \cos (\pi / 4)$ and taking $\chi \approx 0.724$ (see Appendix), we estimated the magnitude of the shear rate for separation to be at least $110 \mathrm{~s}^{-1}$.

\section{DISCUSSION}

For irreversible coagulation occurring at the deep, primary minimum, shear accelerates the coagulation process. Shear can disrupt aggregates as well as bring particles together, however, particularly when the primary particles in aggregates are loosely bonded as a result of reversible aggregation occurring at the second minimum. The key factor here is the strength of particle adhesion, which depends on the surface properties of the particles and also on $\mathrm{NaCl}$ concentration, which changes electrostatic repulsive forces between particles. A brief summary of the information obtained from our tests is as follows:

a

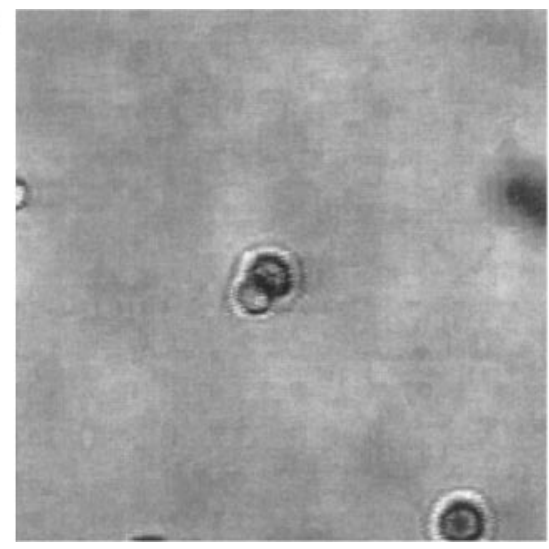

1. At low electrolyte concentration $\left(<0.05 \mathrm{~mol} \cdot \mathrm{L}^{-1} \mathrm{NaCl}\right)$, repulsion between particles prevails over attraction, presenting an energy barrier that prevents further particle approach, and therefore particle adhesion is impossible.

2. At high electrolyte concentration $\left(>0.5 \mathrm{~mol} \cdot \mathrm{L}^{-1} \mathrm{NaCl}\right)$ particle approach leads to fast, strong adhesion as irreversible coagulation occurring at the deep, primary minimum. The second minimum did not appear for particles we used. In contrast with Folkersma's expectation (10) that a shear rate of $1.3 \mathrm{~s}^{-1}$ is sufficient for aggregate (doublets) separation, our experiment showed that shear rates of up to $10.6 \mathrm{~s}^{-1}$ (test 1 ), or even $85 \mathrm{~s}^{-1}$ (according to test 2), are still too small to disrupt aggregates. Therefore, for particles used in our experiment and conditions specified above, it does not seem possible that convection flows (with a shear rate of $1.3 \mathrm{~s}^{-1}$ ) would be able to disrupt the aggregate. Instead, our experiments lead us to expect that shear would actually accelerate coagulation, as long as the shear rate is in the range of our test $\left(<100 \mathrm{~s}^{-1}\right)$, since shear can only increase the frequency of collisions. This idea is consistent with Sun's observation (11) that weak shear flow has negligible influence on

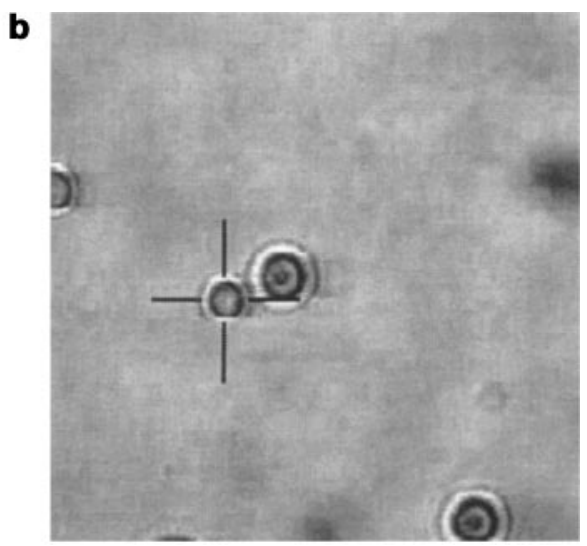

FIG . 5. Separation test for the doublet using optical tweezers under the condition of $0.5 \mathrm{M} \mathrm{NaCl}$ concentration in solution. (a) When the concentration of NaCl was $0.5 \mathrm{M}$, we could not remove the first particle from its partner. (b) The result of the tweezers taking the particle off its partner that rests on the bottom of the cell. 


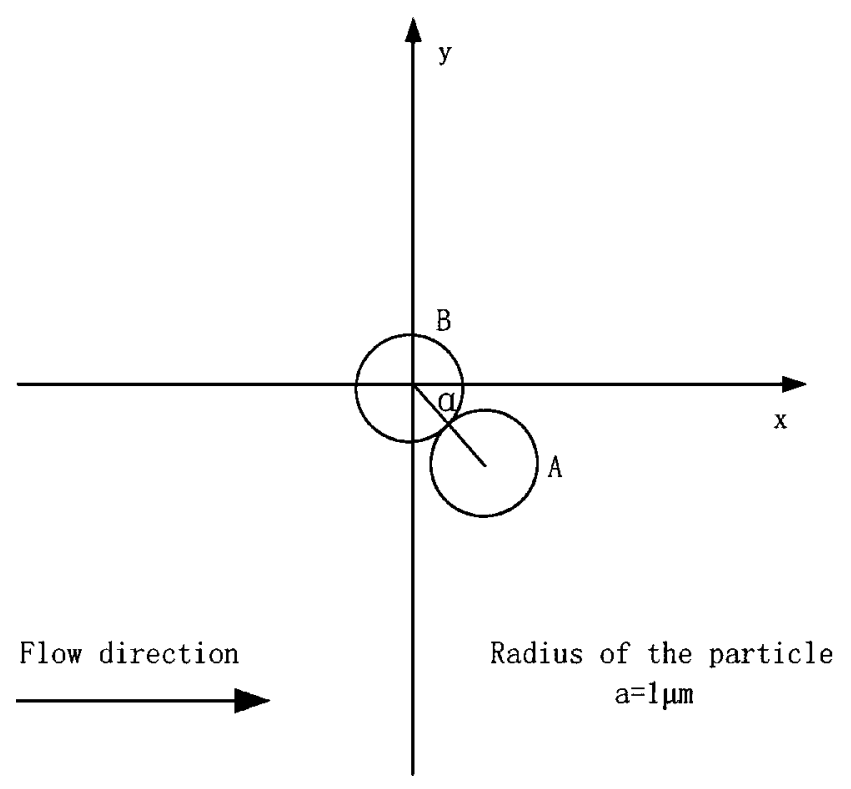

FIG. 6. Schematic representation of two particles, A and B, of Fig. 2 in a uniform flow.

the coagulation. (Shear flow was too weak to make a noticeable difference in their coagulation experiment.)

3. According to test 3 of the laser pulse attack, the depth of the energy minimum of the particle interaction (plus the barrier) should be less than $10^{-10} \mathrm{~J}$. This test can give only an estimate of the upper limit of the energy needed to break the bonds between particles.

4. At medium electrolyte concentration $\left(0.30 \mathrm{~mol} \cdot \mathrm{L}^{-1}\right.$ $\mathrm{NaCl}$ ), the strength of particle adhesion is fairly high but significantly lower than that for the above case. A shear rate magnitude of $110 \mathrm{~s}^{-1}$ (or the corresponding adhesive force between particles of $2 \times 10^{-12} \mathrm{~N}$ ) should be able to disrupt aggregates, according to our test 4 . We could not use higher flow speeds to directly test the disruption of larger aggregates (as done in test 1 ) due to the power limit of our laser.

To minimize the possible influence of the optical trapping force on the coagulation process, all of the aggregates used in tests 1 and 2 were naturally formed. To prepare the aggregates for testing, the coagulation procedure was processed in a separated container for several hours, and then a part of this processed solution was transferred into the sample cell for the test. We cannot exclude the possibility that aggregates connected at the second minimum were broken during the transfer since the connection at the second minimum is very delicate, therefore leaving only aggregates bonded at the primary minimum for our tests.

\section{CONCLUSION}

We have presented an experimental arrangement to estimate adhesive forces between particles within aggregates and also an alternative approach for studying the effect of shear flow on the coagulation process, by directly manipulating individual particles or aggregates. More specifically, our tests 1 and 2 should be able to provide an approximate estimate of an equivalent shear rate for separation of an aggregate. Test 3 can give only an estimate of the upper limit of the energy required to break up the bonds between particles. The accuracy of test 4 would depend on calibration of the trapping force of optical tweezers and here we used the viscous drag to calibrate trapping force because of its simplicity.

Our observations provided us with useful information toward understanding the behavior and process direction of coagulation under certain conditions. As an example, our experiment has provided direct evidence that for the rapid coagulation of $2-\mu \mathrm{m}$ polystyrene latex spheres, shear rates of up to $10.6 \mathrm{~s}^{-1}$ (test 1), or even up to $85 \mathrm{~s}^{-1}$ (according to test 2), are still not enough to disrupt aggregates for particles used in our experiment.

\section{APPE NDIX}

\section{Details of the Hydrodynamic Friction Force Exerted on a Particle in a Moving Liquid}

Here we analyze how the hydrodynamic friction force in a uniform flow was exerted on the considered particle A in test 1 (Fig. 2). When liquid surrounding the aggregate was moving, the whole body of the aggregate rotated (with the rotating center at the trapping center " + ") from the original orientation of Fig. $2 \mathrm{a}$ and then finally stayed at the balanced orientation of Fig. $2 b$. This is different from the case when the aggregate is in the shear flow field because for the latter case the aggregate would keep rotating. Now we consider two particles, A and B, located at the far end of the aggregate in Fig. 2b, schematically presented in Fig. 6. Particle A is attached to the aggregate through particle B at the origin. Following the argument in Ref. (10), the hydrodynamic friction force exerted on particle $\mathrm{A}$ in a liquid moving with a velocity $V$, is $f_{\mathrm{u}}=6 \pi \eta a V \chi$ ( $\chi$ is 0.724 ). We take the angle between the flow direction and the line of centers of particle $A$ and B to be approximately $\pi / 4$, because the most favorable orientation for separation of a pair of particles (the direction of the attractive force keeping the particles together) is different from the hydrodynamic friction force (the direction of the flow) and so a factor of $\cos (\pi / 4)$ should multiply $f_{\mathrm{u}}$. A similar consideration was applied to the case for the shear flow with the shear rate $\gamma$ (10), and the hydrodynamic friction force for separation of particle pairs is $f_{\gamma}=6 \pi \eta a(2 a) \gamma \chi \sin (\pi / 4) \cos (\pi / 4)$. So in our Fig. 2 case of the uniform flow, to yield an equivalent separation force on particle $\mathrm{A}$, we need to have $f_{\mathrm{u}} \approx f_{\gamma}$. In other words, an equivalent shear rate is $\gamma \approx V /$ $(2 a \sin (\pi / 4))$.

Because the aggregate will keep rotating in a shear flow field, the optimal orientation for separation will occur sooner or later. For uniform flow, however, this special orientation has to be artificially manipulated by moving the "trapping center" to hold the proper part of the aggregate, as we did for test 1 . 


\section{ACKNOWLEDGMENTS}

This work was partially supported by Grant 95-Yu-34 from the Ministry of Science and Technology and Grant 19789201 from the National Natural Science Foundation of China (863-512).

\section{REFERENCES}

1. Zeichner, G. R., and Schowalter, W. R., AIChE J. 23, 243 (1977).

2. Firth, B. A., and Hunter, R. J., J. Colloid Interface Sci. 57, 248 (1976).

3. Smith, D. K. M., and Kitchener, J. A., Chem. Eng. Sci. 33, 1631 (1978).

4. van de Ven, T. G. M., and Mason, S. G., Colloid Polym. Sci. 255, 794 (1977).

5. Mühle, K., Colloid Polym. Sci. 8, 660 (1985).

6. Mühle, K., Freiberger Forsh. A720, 22 (1985).

7. Mühle, K., Colloid Surf. 22, 249 (1987).

8. Mühle, K., in "Coagulation and Flocculation: Theory and Application" (B. Dobiáš, Ed.), p. 371. Marcel Dekker Inc., New York, 1993.

9. Folkersma, R., van Diemen, A. J. G., and Stein, H. N., J. Colloid Interface Sci. 206, 482 (1998).
10. Folkersma, R., van Diemen, A. J. G., and Stein, H. N., J. Colloid Interface Sci. 206, 494 (1998).

11. Sun, Z. W., and Qiao, R. L., J. Colloid Interface Sci. 223, 126 (2000).

12. Ashkin, A., Phys. Rev. Lett. 24, 156 (1970).

13. Ashkin, A., Dziedzic, J. M., Brorkholm, J. E., and Chu, S., Opt. Lett. 11, 288 (1986).

14. Block, S. M., Cell Biol. 375 (1990).

15. Crocker, J. C., J. Chem. Phys. 106, 2837 (1997).

16. Crocker, J. C., Matteo, J. A., Dinsmore, A. D., and Yodh, A. G., Phys. Rev. Lett. 82, 4352 (1999).

17. Crocker, J. C., and Grier, D. G., Phys. Rev. Lett. 77, 1897 (1996).

18. Crocker, J. C., and Grier, D. G., Phys. Rev. Lett. 73, 352 (1994).

19. Verma, R., Crocker, J. C., Lubensky, T. C., and Yodh, A. G., Phys. Rev. Lett. 81, 4004 (1998).

20. Bird, R. B., Hassager, O., Armstrong, R. C., and Curtiss, C. F., "Dynamics of Polymetric Liquids," Vol. 2. Wiley, New York, 1977.

21. Stein, H. N., in "The Preparation of Dispersions in Liquids," Vol. 58. Marcel Dekker, New York, 1996.

22. Dai, J., and Sheetz, M. P., in "Methods in Cell Biology: Laser Tweezers in Cell Biology," (Detrich H. W. et al., Eds.) p. 151. Academic Press, San Diego, 1998. 